\title{
Agreement Between Two Procalcitonin Assays in Hospitalized Children
}

\author{
Sophie E. Katz (D) - Laura F. Sartori - Andras Szeles - Rendie McHenry • \\ J. Eric Stanford · Meng Xu • Jennifer M. Colby • Natasha Halasa • \\ Derek J. Williams · Ritu Banerjee
}

Received: May 14, 2019 / Published online: June 29, 2019

(C) The Author(s) 2019

\begin{abstract}
Introduction: Agreement between available procalcitonin (PCT) assays is unclear. We sought to compare concordance between Roche and bioMérieux PCT assays using pediatric samples.

Methods: We evaluated 213 plasma samples from 208 children. We tested each sample on both the Roche and bioMérieux PCT platforms.
\end{abstract}

Enhanced Digital Features To view enhanced digital features for this article go to https://doi.org/10.6084/ m9.figshare.8288603.

S. E. Katz $(\bowtie) \cdot$ A. Szeles · R. McHenry · N. Halasa · R. Banerjee

Division of Pediatric Infectious Diseases, Vanderbilt University Medical Center, Nashville, TN, USA

e-mail: sophie.e.katz@vumc.org

L. F. Sartori

Division of Pediatric Emergency Medicine,

Vanderbilt University Medical Center, Nashville,

TN, USA

J. E. Stanford · J. M. Colby

Department of Pathology, Microbiology and Immunology, Vanderbilt University Medical

Center, Nashville, TN, USA

M. Xu

Department of Biostatistics, Vanderbilt University,

Nashville, TN, USA

D. J. Williams

Division of Pediatric Hospital Medicine, Vanderbilt

University Medical Center, Nashville, TN, USA
Results: At ranges $<2 \mu \mathrm{g} / \mathrm{L}$, the Roche platform had a mean negative bias of $0.13 \mu \mathrm{g} / \mathrm{L}$ versus the bioMérieux platform. This bias resulted in PCT levels that crossed accepted cut points in $12.7 \%$ of patients.

Conclusions: PCT levels measured on either platform are similar, especially at PCT ranges used for antibiotic decision-making algorithms. Funding: This work was supported by an investigator-initiated research agreement through bioMérieux and by the National Institute of Allergy and Infectious Diseases Childhood Infection Research Program (ChIRP), National Institute of Health and the National Center for Advancing Translational Sciences of the National Institute of Health.

Keywords: Method comparison; Pediatrics; Procalcitonin

\author{
Abbreviations \\ PCT Procalcitonin \\ FDA U.S. Food and Drug Administration
}

\section{INTRODUCTION}

Procalcitonin (PCT), a biomarker for bacterial infection, has been evaluated in adults, but is less well-studied in children. In adult patients with bacterial infections, PCT levels have been shown to rise early in infection and fall rapidly 
with effective treatment. In clinical practice, PCT levels above a given cut point suggest bacterial infection that requires antibiotic therapy, while levels below a cut point suggest nonbacterial etiologies that may not require antibiotic therapy. Studies have used a PCT cut point of $0.5 \mu \mathrm{g} / \mathrm{L}$ for sepsis and $0.25 \mu \mathrm{g} / \mathrm{L}$ for pneumonia [1-5]. As most studies have been performed in adults, data regarding the clinical utility of PCT in the pediatric population are limited. Therefore, the ideal PCT cut point in children is not clear. The few existing studies that have evaluated PCT in pediatrics used a variety of PCT cut point values (ranging from 0.13 to $1.75 \mu \mathrm{g} / \mathrm{L}$ ), and these cut points also varied by the site of infection $[6,7]$.

One factor complicating clinical use of PCT is the large number of commercial platforms available for measuring PCT levels, which do not always yield comparable results [5, 8-12]. To date, the majority of pediatric PCT studies have utilized the bioMerieux VIDAS $^{\circledR}$ B.R.A.H.M.S. PCT $^{\mathrm{TM}}$ assay (Marcy-l'Étoile, France), which, until recently, was the only U.S. Food and Drug Administration (FDA)-approved PCT test to guide antibiotic initiation or deescalation decisions. In July 2018, the Roche Elecsys $^{\circledR}$ B.R.A.H.M.S. PCT test (Basel, Switzerland) received FDA approval for the same indication. Therefore, we evaluated agreement between the bioMérieux and Roche PCT assays using samples from hospitalized children with possible infection. We sought to determine the bias between assays and how often clinical decision making could be impacted by using one test versus the other.

\section{METHODS}

\section{Sample Collection, Processing and Procalcitonin Testing}

We conducted a single-center cross-sectional study using plasma samples collected from children (less than 19 years of age) presenting to an urban, 267-bed pediatric tertiary care hospital. We included two cohorts of children. Cohort 1 consisted of 142 stored plasma specimens, collected as part of an earlier study from
December 2014 through January 2017 from children presenting to the emergency department with clinical and radiographic evidence of community-acquired pneumonia [13]. Subjects were excluded if they met any of the following criteria: (1) alternative non-pneumonia diagnosis, (2) hospitalized within the previous 7 days (including hospital transfers), (3) primary or secondary immunocompromising conditions, (4) presence of tracheostomy, (5) diagnosis of cystic fibrosis, or (6) extended or longterm care facility resident.

Cohort 2 was comprised of an additional 71 stored plasma specimens from children presenting to either the emergency department or the oncology clinic for whom a blood culture was ordered due to concerns for sepsis, and who were prospectively enrolled from November 2017 through June 2018. Subjects were excluded if they met any of the following criteria: (1) neonates younger than 7 days of age, (2) infants who were ultimately admitted to the neonatal intensive care unit, or (3) the participant, parent or guardian did not provide informed consent and/or assent, when appropriate.

Samples for PCT testing for patients in Cohort 1 were collected in a lithium heparin gel tube at the time of study enrollment and refrigerated for a maximum of $12 \mathrm{~h}$ prior to centrifugation and storage of plasma at $-80^{\circ} \mathrm{C}$ until PCT analysis. Frozen samples were thawed and separated into two aliquots. One aliquot was then run on the bioMérieux platform, while the other aliquot underwent an additional freeze-thaw cycle prior to testing on the Roche platform. For Cohort 2, blood samples were obtained in a lithium heparin gel tube for PCT analysis at the time that their initial blood culture was drawn. These samples were refrigerated within $3 \mathrm{~h}$ of collection, centrifuged, separated into two aliquots within $72 \mathrm{~h}$ of collection, and the plasma was then frozen at $-80{ }^{\circ} \mathrm{C}$ until PCT analysis. Each aliquot was only tested on one platform. Testing generally occurred within 3 months of specimen collection.

Procalcitonin testing was performed using the bioMérieux $\operatorname{VIDAS}^{\circledR} 3$ platform and the Roche Elecsys ${ }^{\circledR}$ BRAHMS PCT reagent on an e411 immunoassay analyzer. The minimum volume of serum required for testing was $0.2 \mathrm{~mL}$ 
on the bioMerieux assay and $0.5 \mathrm{~mL}$ for the Roche assay. The reportable range of each assay is $0.05-200 \mu \mathrm{g} / \mathrm{L}$ for the bioMerieux assay and $0.02-100 \mu \mathrm{g} / \mathrm{L}$ for the Roche assay. Aliquots from all samples were tested on both platforms. For both cohorts, treating providers were not aware of the PCT results, which were not reported in the electronic medical record.

\section{Statistical Analyses}

Pearson's correlation, simple linear regression and Bland-Altman plots were used to compare performance of the two PCT platforms. All analyses were performed using R v.3.5 (Boston, MA, USA) or Stata/IC v.15.1 for Mac (College Station, TX, USA). $P$ values $<0.05$ were considered statistically significant. We conducted a subgroup analysis comparing values from Cohort 1 and Cohort 2 separately, to account for the additional freeze-thaw cycle that samples from Cohort 1 underwent prior to testing on the Roche platform.

\section{Compliance with Ethics Guidelines}

The study procedures were approved by the Vanderbilt University Institutional Review Board, reference number 171094. The study conformed with the Helsinki Declaration of 1964, as revised in 2013, concerning human and animal rights, and Springer's policy concerning informed consent was followed. Written informed consent for study participation and publication of de-identified results was obtained from the parents or guardians of the children who served as participants in the investigation and, when appropriate, assent from the subjects themselves was obtained.

\section{RESULTS}

During the study period, 208 unique patients contributed 213 specimens, with 142 (67\%) specimens from Cohort 1 and 71 (33\%) specimens from Cohort 2. The median age of the 208 patients was 5.2 years (range 8 days-18.9 years),
157 (74\%) were white, 164 (77\%) were nonHispanic or Latino, and 137 (65\%) were male.

The Roche and bioMérieux tests displayed excellent correlation across the entire range of PCT concentrations (Pearson's correlation coefficient 0.95 , line of best fit equation $y=-0.27+0.64 x ;$ Fig. 1a). In our subgroup analysis, there was excellent correlation among PCT values from Cohort 1 (Pearson's correlation coefficient 0.98 , line of best fit equation $y=$ $-0.34+0.66 x)$, and good correlation among PCT values from Cohort 2 Pearson's correlation coefficient 0.78 , line of best fit equation $y=0.17+0.47 x)$. Platform concordance was better at lower values than at higher values (Fig. 1b, c). The Roche platform resulted in lower PCT values as compared to the bioMérieux platform across the entire range of PCT values, with a mean bias of $0.13 \mu \mathrm{g} / \mathrm{L}$ in the range $<2 \mu \mathrm{g} / \mathrm{L}$, where accepted cut points for PCT-guided antibiotic treatment decisions lie (Fig. 1b, c). However, this bias did not always lead to a clinically significant difference (i.e., crossing a PCT cut point). That is, only 10 samples (4.7\% of 213$)$ had PCT levels that were $0.25 \mu \mathrm{g} / \mathrm{L}$ or higher on the bioMérieux platform, but less than $0.25 \mu \mathrm{g} / \mathrm{L}$ on the Roche platform. Similarly, 16 samples (7.5\% of 213 ) had PCT levels of $0.5 \mu \mathrm{g} / \mathrm{L}$ or higher on the bioMérieux platform, but less than $0.5 \mu \mathrm{g} / \mathrm{L}$ on the Roche platform. One sample was greater than $0.5 \mu \mathrm{g} / \mathrm{L}$ on the Roche platform, but less than $0.5 \mu \mathrm{g} / \mathrm{L}$ on the bioMerieux platform. Overall, this resulted in a total of 27 samples (12.7\%) in which the measurement bias would have potentially altered antibiotic therapy decisions.

\section{DISCUSSION}

In this single-center study using stored specimens from children with possible sepsis or pneumonia, the bioMérieux and Roche PCT tests demonstrated similar results, with excellent concordance, particularly at levels below $2 \mu \mathrm{g} / \mathrm{L}$ where cut points for antibiotic decision making algorithms lie (ranging from 0.13 to $1.75 \mu \mathrm{g} / \mathrm{L}$ in prior studies) $[6,7]$. The diagnostic utility of PCT in children with sepsis is unclear, and further studies are warranted to determine 



whether cut points used in adults also apply to children. The accepted PCT cut point for adults with sepsis of $0.5 \mu \mathrm{g} / \mathrm{L}$ and for adults and children with pneumonia of $0.25 \mu \mathrm{g} / \mathrm{L}$ are well within the range where the platforms demonstrate good agreement [1, 2, 11, 14, 15]. Our data demonstrate that PCT levels within the range of $0-2 \mu \mathrm{g} / \mathrm{L}$ are approximately $0.13 \mu \mathrm{g} / \mathrm{L}$ lower using the Roche test as compared to the bioMérieux test. Although this difference would have only impacted approximately $13 \%$ of samples in our study, clinicians should be aware of the potential difference in PCT measurements, especially when a patient is transferred between two institutions that use different PCT
4Fig. 1 Comparison of PCT measurement by bioMérieux and Roche Platforms. a Scatterplot of PCT values measured by each platform. Combined data from Cohort 1 and Cohort 2 are shown. Blue line represents regression line and black line represents line of unity. Black triangles represent PCT values from Cohort 1 and red circles represent PCT values from Cohort 2. Graph restricted to values $\leq 100 \mu \mathrm{g} / \mathrm{L}$ for better visualization. b Pair plot of PCT values measured by each platform, restricted to values $\leq 2 \mu \mathrm{g} / \mathrm{L}$. Each pair represents one patient's plasma sample. Solid line represents clinically accepted cut point for sepsis, dashed line represents clinically accepted cut point for pneumonia. Asterix represents pair of samples that cross clinically accepted cut points. c Bland-Altman plot of PCT values, restricted to PCT values $\leq 2 \mu \mathrm{g} / \mathrm{L}$. Horizontal dashed black lines represent the mean of the difference between bioMérieux and Roche values, and the upper and lower limits of the $95 \%$ confidence interval. Black triangles represent PCT values from Cohort 1 and red circles represent PCT values from Cohort 2 . The mean of the difference is approximately $0.13 \mu \mathrm{g} / \mathrm{L}$, indicating that the mean PCT measured on the bioMérieux platform is $0.13 \mu \mathrm{g} / \mathrm{L}$ higher than the mean PCT measured on the Roche platform. Vertical solid black lines denote clinically accepted cut points; above $0.5 \mu \mathrm{g} / \mathrm{L}$ suggests bacterial infection is likely, and below $0.25 \mu \mathrm{g} / \mathrm{L}$ suggests bacterial infection is unlikely. PCT procalcitonin

platforms. Assays that use the B.R.A.H.M.S. PCT monoclonal antibodies, such as the Roche and bioMérieux assays, are expected to have a high level of concordance, as was seen in this study. Notably, there are other PCT assays entering the market that do not use these antibodies, and it remains uncertain whether these assays will demonstrate the same degree of concordance $[10,16]$.

Our study has a number of strengths, including a large sample size and comparison of two commonly used and commercially available platforms. However, it also has some limitations. We did not include the gold-standard platform for testing, the BRAHMS PCT Kryptor system (Hennigsdorf, Germany) [10]. Additionally, due to logistical issues, the samples that were stored frozen for longer than 6 months underwent an additional freeze-thaw cycle prior to testing on the Roche platform. It is 
possible that this additional freeze-thaw cycle impacted the Roche PCT test results and potentially contributed to the observed bias between the two methods, although a prior study demonstrated that repeated freeze-thaw cycles do not have significant influence on measured PCT levels [17]. We used stored samples for PCT measurement, which have been shown to demonstrate a modest decline of approximately $10 \%$ after deep-frozen storage for 3-5 years [18]. Our study compared Roche and bioMérieux test results from the same blood sample, so long-term storage of samples should not account for differences between platforms.

\section{CONCLUSIONS}

We conclude that PCT levels measured on the bioMérieux and Roche platforms are similar, especially at ranges $<2 \mu \mathrm{g} / \mathrm{L}$, where cut points lie for antibiotic decision-making algorithms. The Roche assay yielded lower PCT values than the bioMérieux assay, producing a bias that would have potentially altered PCT interpretation in a modest percentage of patients. As PCT is increasingly used for patient care, clinicians should be aware that PCT measurements can differ across platforms, although differences appear small and are unlikely to have major clinical impact.

\section{ACKNOWLEDGEMENTS}

Funding. This work was supported by an investigator-initiated research agreement through bioMérieux and by the National Institute of Allergy and Infectious Diseases Childhood Infection Research Program (ChIRP), National Institute of Health and the National Center for Advancing Translational Sciences of the National Institute of Health. This content is solely the responsibility of the authors and does not necessarily represent the official views of the National Institutes of Health. All authors had full access to all of the data in this study and take complete responsibility for the integrity of the data and the accuracy of the data analysis.

Authorship. All named authors meet the International Committee of Medical Journal Editors (ICMJE) criteria for authorship for this article, take responsibility for the integrity of the work as a whole, and have given their approval for this version to be published.

Disclosures. We are grateful to bioMérieux for providing PCT test kits, reagents and a bioMérieux VIDAS $^{\circledR} 3$ instrument. The company was not involved in study design or conduct, data analysis or manuscript preparation or publication. Sophie Katz received investigatorinitiated research funding from bioMérieux and Roche. Ritu Banerjee received investigator-initiated research funding from bioMérieux and Roche. Laura Sartori, Andras Szeles, Rendie McHenry, J. Eric Stanford, Meng Xu, Jennifer Colby, Natasha Halasa and Derek Williams have nothing to disclose.

Compliance with Ethics Guidelines. The study procedures were approved by the Vanderbilt University Institutional Review Board (IRB), reference number 171094. The study conformed with the Helsinki Declaration of 1964, as revised in 2013, concerning human and animal rights, and Springer's policy concerning informed consent was followed. Written informed consent for study participation and publication of results was obtained from the parents or guardians of the children who served as participants in the investigation and, when appropriate, assent from the subjects themselves was obtained.

Data Availability. The datasets generated and/or analyzed during the current study are available from the corresponding author on reasonable request. We thank the participants of the study.

Open Access. This article is distributed under the terms of the Creative Commons Attribution-NonCommercial 4.0 International License (http://creativecommons.org/licenses/ by-nc/4.0/), which permits any non- 
commercial use, distribution, and reproduction in any medium, provided you give appropriate credit to the original author(s) and the source, provide a link to the Creative Commons license, and indicate if changes were made.

\section{REFERENCES}

1. Bouadma L, Luyt CE, Tubach F, et al. Use of procalcitonin to reduce patients' exposure to antibiotics in intensive care units (PRORATA trial): a multicentre randomised controlled trial. Lancet. 2010;375(9713):463-74.

2. de Jong E, van Oers JA, Beishuizen A, et al. Efficacy and safety of procalcitonin guidance in reducing the duration of antibiotic treatment in critically ill patients: a randomised, controlled, open-label trial. Lancet Infect Dis. 2016;16(7):819-27.

3. Schuetz P, Christ-Crain M, Thomann R, et al. Effect of procalcitonin-based guidelines vs standard guidelines on antibiotic use in lower respiratory tract infections: the ProHOSP randomized controlled trial. JAMA. 2009;302(10):1059-66.

4. Huang DT, Yealy DM, Filbin MR, et al. Procalcitonin-guided use of antibiotics for lower respiratorytract infection. $N$ Engl J Med. 2018;379(3): 236-49.

5. Esposito S, Tagliabue C, Picciolli I, et al. Procalcitonin measurements for guiding antibiotic treatment in pediatric pneumonia. Respir Med. 2011;105(12):1939-45.

6. Maniaci V, Dauber A, Weiss S, Nylen E, Becker KL, Bachur R. Procalcitonin in young febrile infants for the detection of serious bacterial infections. Pediatrics. 2008;122(4):701-10.

7. Downes KJ, Weiss SL, Gerber JS, et al. A pragmatic biomarker-driven algorithm to guide antibiotic use in the pediatric intensive care unit: the optimizing antibiotic strategies in sepsis (OASIS) study. J Pediatr Infect Dis Soc. 2017;6(2):134-41.

8. Milcent K, Faesch S, Gras-Le Guen C, et al. Use of procalcitonin assays to predict serious bacterial infection in young febrile infants. JAMA Pediatr. 2016;170(1):62-9.
9. Baer G, Baumann P, Buettcher M, et al. Procalcitonin guidance to reduce antibiotic treatment of lower respiratory tract infection in children and adolescents (ProPAED): a randomized controlled trial. PLoS ONE. 2013;8(8):e68419.

10. Dipalo M, Guido L, Micca G, et al. Multicenter comparison of automated procalcitonin immunoassays. Pract Lab Med. 2015;2:22-8.

11. Stockmann C, Ampofo K, Killpack J, et al. Procalcitonin accurately identifies hospitalized children with low risk of bacterial community-acquired pneumonia. J Pediatr Infect Dis Soc. 2018;7(1): 46-53.

12. Chambliss AB, Hayden J, Colby JM. Evaluation of procalcitonin immunoassay concordance near clinical decision points. Clin Chem Lab Med. 2019. https://doi.org/10.1515/cclm-2018-1362.

13. Sartori L, Katz S, Grijalva CG, et al. Clinical utility of procalcitonin in children hospitalized with community-acquired pneumonia. In: Poster presented at: Pediatric Academic Societies annual meeting 2018; Toronto, Canada.

14. Schuetz P, Briel M, Christ-Crain M, et al. Procalcitonin to guide initiation and duration of antibiotic treatment in acute respiratory infections: an individual patient data meta-analysis. Clin Infect Dis. 2012;55(5):651-62.

15. Christ-Crain M, Jaccard-Stolz D, Bingisser R, et al. Effect of procalcitonin-guided treatment on antibiotic use and outcome in lower respiratory tract infections: cluster-randomised, single-blinded intervention trial. Lancet. 2004;363(9409):600-7.

16. Ceriotti F, Marino I, Motta A, Carobene A. Analytical evaluation of the performances of Diazyme and BRAHMS procalcitonin applied to Roche Cobas in comparison with BRAHMS PCT-sensitive Kryptor. Clin Chem Lab Med. 2017;56(1):162-9.

17. Meisner M, Tschaikowsky K, Schnabel S, Schmidt J, Katalinic A, Schuttler J. Procalcitonin-influence of temperature, storage, anticoagulation and arterial or venous asservation of blood samples on procalcitonin concentrations. Eur J Clin Chem Clin Biochem. 1997;35(8):597-601.

18. Schuetz P, Christ-Crain M, Huber AR, Muller B. Longterm stability of procalcitonin in frozen samples and comparison of Kryptor and VIDAS automated immunoassays. Clin Biochem. 2010;43(3):341-4. 